\title{
FATORES MOTIVACIONAIS DE JOVENS ATLETAS DE VÔLEI
}

ESP. LIVIA TAVARES DA SILVA CAMPOS

Especialista em Treinamento Desportivo (EEFD - UFRJ)

(Rio de Janeiro - Rio de Janeiro - Brasil)

E-mail: liviafla@gmail.com

\section{DRA. SÍLVIA MARIA AGATTI LÜRDOF}

Professora Assistente (UFRJ)

Coordenadora do Núcleo de Estudos Sociocorporais e

Pedagógicos em EF e Esportes (NESPEFE/EEFD - UFRJ)

Doutorado em Educação (UFRJ) (Rio de Janeiro - Rio de Janeiro -Brasil)

E-mail: sagatti.rlk@terra.com.br

\section{RESUMO}

O estudo objetivou identificar o quanto os fatores motivacionais determinam a permanência de jovens atletas no voleibol de alto nível e compará-los quanto ao gênero e categoria. A coleta de dados foi realizada por meio da aplicação do questionário de Escala de Motivos para a Prática Esportiva (EMPE) a 96 jovens atletas de vôlei de quadra (46 meninas e 50 meninos). Os fatores que mais motivaram os atletas para a prática do vôlei foram: aperfeiçoamento técnico, saúde e afliação. Na comparação quanto ao gênero, com exceção dos fatores saúde e status, as atletas apresentaram maiores índices de motivação do que os atletas. Comparando os fatores motivacionais segundo a categoria, todos os itens analisados, exceto afiliação, motivam da mesma forma os atletas.

PALAVRAS-CHAVE: Motivação; treinamento juvenil; vôlei de quadra; jovens atletas. 


\section{INTRODUÇÃO}

O esporte moderno, com sua organização estrutural, investimentos milionários e publicidade globalizada, alcançou uma dimensão extraordinária, fazendo-se presente na vida de milhões de pessoas em todas as regiões do planeta. $O$ apelo à prática da atividade desportiva encontra especial ressonância entre as camadas mais jovens das populações, seja pela sua crucial importância nos processos de educação e de desenvolvimento físico, psicológico e social infantil e juvenil, seja pelo intensivo direcionamento da mídia, face à expressão da parcela de mercado que tais faixas de idade representam.

Weiberg e Gould (200 I) estimam em aproximadamente 25 milhões o número de crianças praticantes de pelo menos uma atividade desportiva. Há de se considerar, nesse quadro, que a prática de esportes, em suas diferentes modalidades, exige de crianças e adolescentes, para a permanência no processo de preparação e competição desportiva, um elevado nível de desenvolvimento de suas funções, qualidades e estados psíquicos (BENCK; CASAL, 2006).

A prática da atividade desportiva é, essencialmente, dirigida para a aplicação do sentido e da intensidade dos esforços com vistas ao alcance de metas específicas. Essa ação decorre de um motivo, um impulso, uma intenção, que leva o indivíduo a agir de determinada forma (MAGILL, 1984). Davidoff (200 I) refere-se a motivo ou motivação como um estado interno que resulta de uma necessidade que ativa ou desperta um comportamento usualmente dirigido ao cumprimento da necessidade ativante.

Motivação é um termo que alcança qualquer comportamento que se dirija a um objetivo; inicia-se com um motivo e provoca uma ação focada para a realização de metas específicas. Para Samulski (2002), o estudo da motivação tenta compreender porque um indivíduo, num certo tempo, escolhe uma forma de comportamento e a realiza com determinada intensidade e persistência, uma vez que a motivação para a prática desportiva depende da interação entre a personalidade (expectativas, interesses, motivos, necessidades) e fatores do meio ambiente, como facilidade, tarefas atraentes, desafios e influências sociais. A motivação, nesse sentido, é o resultado de um somatório de fatores pessoais e ambientais, em um processo intencional e interior ao indivíduo. A interação dos fatores forma uma decisão que impulsiona o indivíduo a alcançar a necessidade ativante, podendo não ser motivado de forma semelhante em outras situações (KNIJNIK; GREGUOL; SANTOS, 200 I). É devido ao nível de motivação para determinada meta que os indivíduos escolhem uma tarefa específica, executando-a com maior empenho do que se estivessem fazendo tarefa distinta (SAMULSKI, 2002). 
Conhecer os motivos que impulsionam as crianças e adolescentes à prática de uma modalidade de desporto pode vir a aperfeiçoar as atividades a serem desenvolvidas durante o treinamento, levando-as a melhorarem o seu desempenho e, com isso, sentirem-se mais motivadas. A motivação por sua vez pode gerar melhora de desempenho, num círculo virtuoso absolutamente desejável. Mourouço (2007) descreve que a motivação é responsável pela direção, intensidade e persistência dos indivíduos numa determinada modalidade esportiva e manisfesta-se como principal fonte de êxito esportivo, devendo ser mantida permanentemente. $\bigcirc$ estudo sobre como os fatores motivacionais impulsionam crianças e adolescentes a se envolverem na prática de esportes pode ser ferramenta de grande utilidade para a elaboração de treinos estratégicos, auxiliando o processo de ensino-aprendizagem. Para Berleze, Vieira e Krebs (2002) que o conhecimento sobre elementos motivadores ajuda em planejamentos mais direcionados ao interesse do praticante, aumentando a probabilidade de permanência na prática da atividade desportiva. Alvarez (2006), ao estudar o abandono do esporte por jovens atletas, acrescenta ainda que as atividades fora do esporte também são importante no planejamento da carreira esportiva desses meninos e meninas.

Em face da relevância do tema, este estudo tem o objetivo de identificar quanto os fatores motivacionais influenciam crianças e adolescentes a permanecerem praticando o voleibol de alto nível. Pretende-se, ainda, estabelecer comparações entre as categorias e sexo para verificar eventuais particularidades que possam influenciar na motivação dos jovens atletas.

\section{MATERIAIS E MÉTODOS}

A amostra deste estudo é composta por 96 atletas (46 meninas e 50 meninos) de um clube de grande porte, localizado na zona sul da cidade do Rio de Janeiro, que costuma revelar atletas para as seleções estaduais e brasileiras. Esta agremiação participa em todas as categorias de base dos campeonatos promovidos pela Federação de Vôlei do Rio de Janeiro, além de participar de torneios nacionais entre clubes.

Como critério para inclusão no estudo, todos os voluntários deveriam ser vinculados obrigatoriamente à Federação de Vôlei do Rio de Janeiro, pertencendo, em 2009, ao clube onde os dados foram coletados.

Com o intuito de estudar as diferenças motivacionais entre os atletas segundo o sexo e a categoria (mirim, infantil, infanto ou juvenil), a amostra foi dividida em 8 grupos, conforme é demonstrado na Tabela I. 
Tabela I - Número de Participantes por categoria em percentual

\begin{tabular}{|c|c|c|c|c|c|}
\hline & $\begin{array}{l}\text { Mirim } \\
(\mathrm{n} / \%)\end{array}$ & $\begin{array}{l}\text { Infantil } \\
(\mathrm{n} / \%)\end{array}$ & $\begin{array}{l}\text { Infanto } \\
(\mathrm{n} / \%)\end{array}$ & $\begin{array}{l}\text { Juvenil } \\
(\mathrm{n} / \%)\end{array}$ & $\begin{array}{l}\text { Total } \\
(\mathrm{n} / \%)\end{array}$ \\
\hline Feminino & $15 / 32,6$ & $|8 / 39|$, & $11 / 23,9$ & $02 / 4,3$ & $46 / 100,0$ \\
\hline Masculino & $14 / 28,0$ & $15 / 30,0$ & I I/ 22,0 & $10 / 20,0$ & $50 / 100,0$ \\
\hline Total & $29 / 30,2$ & $33 / 34,4$ & $22 / 22,9$ & $12 / 12,5$ & $96 / 100,0$ \\
\hline
\end{tabular}

Cabe destacar que ao comparar os atletas segundo a categoria, está sendo comparada também a faixa etária, já que o ano de nascimento é o que determina a qual categoria esses atletas pertencem (Quadro I).

\begin{tabular}{|l|l|}
\hline Ano de Nascimento & Categoria \\
\hline A partir de 1996 & Mirim \\
\hline 1995 ou 1994 & Infantil \\
\hline 1993 ou 1992 & Infanto \\
\hline 1991, 1990 ou 1989 & Juvenil \\
\hline
\end{tabular}

Quadro I - Relação entre a categoria dos atletas e o ano de nascimento

A coleta de dados foi realizada através de um questionário, onde havia campos específicos para que os participantes informassem sua categoria e seu sexo. Os dados relativos aos fatores motivacionais foram coletados através da Escala de Motivos para a Prática Esportiva (EMPE), versão traduzida e validada para o Brasil por Barroso (2007) do Participation Motivation Questionnaire (PMQ) de Gill, Gross e Huddleston (1993). Este questionário é composto por 33 perguntas, que devidamente agrupadas identificam sete fatores de motivação para a prática esportiva: Status, Condicionamento Físico, Liberação de Energia, Contexto, Aperfeiçoamento Técnico, Afiliação e Saúde. Os voluntários atribuem um valor de zero a I 0 (escala Likert de I I pontos) para cada pergunta de acordo com o quanto este fator o motiva a continuar praticando o vôlei de quadra competitivo.

A análise exploratória dos dados se deu através do calculo da Mediana, Média, Desvio Padrão, Valor Mínimo e Valor Máximo, das sete categorias de motivação. Os testes utilizados foram não-paramétricos devido às características das variáveis do estudo.

O teste $U$ de Mann-Whitney foi utilizado para comparação dos fatores motivacionais segundo sexo, uma vez que os participantes do estudo foram divididos em dois grupos. Para a comparação destes fatores segundo a categoria foi utilizado 
o teste de Krustal-Wallis, pelo fato dos participantes estarem divididos em mais de dois grupos (mirim, infantil, infanto e juvenil).

As análises estatísticas foram realizadas no software SPSS 13.0. O nível de significância adotado foi de 5\% ( $p \leq 0,05)$.

Os procedimentos empregados na pesquisa estão de acordo com os critérios éticos estabelecidos nos termos da Resolução 196/96 e 25।/97 do Conselho Nacional de Saúde.

\section{RESULTADOS}

Os fatores que, segundo a média, mais motivaram os atletas foram, pela ordem: Aperfeiçoamento Técnico $(9,25 \pm 1,42)$, Saúde $(8,67 \pm 2,27)$, Afiliação $(8,0 \pm 1,82)$, Condicionamento Físico $(7,67 \pm 1,43)$, Status $(7,86 \pm 1,90)$, Liberação de Energia $(7,17 \pm 2,04)$ e Contexto $(7,00 \pm 2,27)$, conforme pode ser observado na Tabela 2.

Tabela 2 - Mediana, Média, Desvio Padrão, Valor Mínimo e Valor

Máximo dos Fatores Motivacionais dos Participantes do Estudo $(n=96)$

\begin{tabular}{l|llllll}
\hline & Fatores Motivacionais & Mediana & Média & $\begin{array}{c}\text { Desvio } \\
\text { Padrão }\end{array}$ & $\begin{array}{c}\text { Valor } \\
\text { Mínimo }\end{array}$ & Valor Máximo \\
\hline 1 & Status & 7,86 & 7,55 & 1,90 & 3,00 & 10,00 \\
2 & Cond. Físico & 7,67 & 7,64 & 1,94 & 2,00 & 10,00 \\
3 & Lib. de Energia & 7,17 & 6,90 & 2,04 & 1,00 & 10,00 \\
4 & Aperf. Técnico & 9,25 & 8,70 & 1,42 & 4,00 & 10,00 \\
5 & Afiliação & 8,00 & 7,79 & 1,82 & 2,00 & 10,00 \\
6 & Saúde & 8,67 & 7,85 & 2,27 & 0 & 10,00 \\
\hline 7 & Contexto & 7,00 & 6,42 & 2,27 & 0 & 10,00 \\
\hline
\end{tabular}

Ao comparar meninos e meninas foi constatado que, conforme é mostrado na Tabela 3, há diferença estatística significativa entre os grupos para os fatores motivacionais Condicionamento Físico, Liberação de Energia, Aperfeiçoamento Técnico, Afiliação e Contexto. Os fatores Saúde e Status foram os únicos itens que motivam da mesma forma os atletas, independentemente do sexo. 
Tabela 3 - Comparação dos Fatores Motivacionais dos Participantes segundo Gênero

\begin{tabular}{|c|c|c|c|c|}
\hline & \multirow[t]{2}{*}{ Fatores Motivacionais } & \multicolumn{2}{|c|}{ Média e Desvio Padrão } & \multirow{2}{*}{ Valor de $\mathrm{p}$} \\
\hline & & Feminino $(n=46)$ & Masculino $(n=50)$ & \\
\hline 1 & Status & $7,73 \pm 2,07$ & $7,39 \pm 2,45$ & 0,193 \\
\hline 2 & Condicionamento Físico & $8,06 \pm 1,86$ & $7,26 \pm 1,73$ & $0,035^{*}$ \\
\hline 3 & Liberação de Energia & $7,55 \pm 2,02$ & $6,31 \pm 1,89$ & $0,00 I^{*}$ \\
\hline 4 & Aperfeiçoamento Técnico & $9,07 \pm 1,27$ & $8,37 \pm 1,48$ & $0,005^{*}$ \\
\hline 5 & Afiliação & $8,67 \pm 1,43$ & $6,98 \pm 1,77$ & $0,000 *$ \\
\hline 6 & Saúde & $8,20 \pm 2,08$ & $7,53 \pm 2,40$ & 0,116 \\
\hline 7 & Contexto & $6,96 \pm 2,34$ & $5,93 \pm 2,11$ & $0,0|| *$ \\
\hline
\end{tabular}

*Mann-Whitney Test; $p \leq 0,05$

Ao comprar os sete fatores motivacionais em relação à categoria foi observado que seis destes fatores (Status, Condicionamento Físico, Liberação de Energia, Aperfeiçoamento Técnico, Saúde e Contexto) motivam da mesma forma os atletas independentemente da categoria a que pertencem. Apenas o fator Afiliação apresentou diferença estatística significativa entre as categorias mirim X infanto e mirim X juvenil (Tabela 4).

Tabela 4 - Comparação dos Fatores Motivacionais dos Participantes segundo Categoria

\begin{tabular}{|c|c|c|c|c|c|c|}
\hline & \multirow{2}{*}{$\begin{array}{c}\text { Fatores } \\
\text { Motivacionais }\end{array}$} & \multicolumn{4}{|c|}{ Média e Desvio Padrão } & \multirow[b]{2}{*}{ Valor de $p$} \\
\hline & & $\begin{array}{l}\text { Mirim } \\
(n=29)\end{array}$ & $\begin{array}{l}\text { Infantil } \\
(n=33)\end{array}$ & $\begin{array}{l}\text { Infanto } \\
(n=22)\end{array}$ & $\begin{array}{l}\text { Juvenil } \\
(n=12)\end{array}$ & \\
\hline I & Status & $7,83 \pm 1,84$ & $7,7 \mid \pm 1,85$ & $6,83 \pm 2,10$ & $7,75 \pm 1,65$ & 0,307 \\
\hline 2 & Cond. Físico & $7,79 \pm 1,97$ & $7,42 \pm 2,13$ & $7,61 \pm 1,97$ & $7,94 \pm 1,32$ & 0,877 \\
\hline 3 & Lib. de Energia & $7,28 \pm 1,81$ & $6,99 \pm 2,13$ & $6,12 \pm 2,33$ & $7,18 \pm 1,50$ & 0,333 \\
\hline 4 & Aperf. Técnico & $9,16 \pm 0,95$ & $8,67 \pm|, 5|$ & $8,23 \pm 1,58$ & $8,54 \pm 1,64$ & 0,163 \\
\hline 5 & Afiliação & $8,46 \pm 1,31$ & $7,75 \pm 2,15$ & $7,17 \pm 1,88^{*}$ & $7,40 \pm 1,32^{*}$ & $0,045^{*}$ \\
\hline 6 & Saúde & $8,37 \pm 1,77$ & $7,43 \pm 2,69$ & $7,52 \pm 2,36$ & $8,36 \pm 1,75$ & 0,543 \\
\hline 7 & Contexto & $7,10 \pm 1,90$ & $6,62 \pm 2,30$ & $5,46 \pm 2,50$ & $6,00 \pm 2,14$ & 0,091 \\
\hline
\end{tabular}

*Kruskall-Wallis; $p \leq 0,05$

$¥$ Mann-Whitney: mirim $\times$ infanto; $p=0,0 \mid 4$; mirim $X$ juvenil; $p=0,012$

Foi possível ainda constatar que independentemente da categoria ou gênero, o fator motivacional Aperfeiçoamento Técnico foi considerado o mais importante em todos os casos (Tabelas 3 e 4). 
A Tabela 5 revela que, levando em consideração apenas atletas do sexo feminino, também não há diferença estatística significativa ao comparar os sete fatores motivacionais segundo a categoria.

Tabela 5 - Comparação dos Fatores Motivacionais segundo Categoria - Gênero Feminino

\begin{tabular}{|c|c|c|c|c|c|c|}
\hline & \multirow{2}{*}{$\begin{array}{c}\text { Fatores } \\
\text { Motivacionais }\end{array}$} & \multicolumn{4}{|c|}{ Média e Desvio Padrão } & \multirow[b]{2}{*}{ Valor de $p$} \\
\hline & & $\begin{array}{l}\text { Mirim } \\
(n=15)\end{array}$ & $\begin{array}{l}\text { Infantil } \\
(n=\mid 8)\end{array}$ & $\begin{array}{l}\text { Infanto } \\
(n=\mid I)\end{array}$ & $\begin{array}{l}\text { Juvenil } \\
(n=2)\end{array}$ & \\
\hline I & Status & $8,02 \pm 1,95$ & $8,33 \pm 1,62$ & $6,27 \pm 2,50$ & $8,21 \pm 0,91$ & 0,160 \\
\hline 2 & Cond. Físico & $7,96 \pm 1,86$ & $8,67 \pm 1,45$ & $7,39 \pm 2,37$ & $7,00 \pm 0,94$ & 0,339 \\
\hline 3 & Lib. de Energia & $7,86 \pm 1,43$ & $7,99 \pm 1,86$ & $6,29 \pm 2,68$ & $8,25 \pm 0,35$ & 0,270 \\
\hline 4 & Aperf. Técnico & $9,52 \pm 0,47$ & $9,31 \pm 0,91$ & $7,95 \pm 1,93$ & $9,63 \pm 0,18$ & 0,175 \\
\hline 5 & Afiliação & $9,08 \pm 0,69$ & $8,91 \pm 1,48$ & $7,91 \pm 1,81$ & $7,50 \pm 1,56$ & 0,117 \\
\hline 6 & Saúde & $8,42 \pm 2,03$ & $8,54 \pm 1,84$ & $7,30 \pm 2,58$ & $8,50 \pm 1,18$ & 0,595 \\
\hline 7 & Contexto & $7,31 \pm 1,72$ & $7,69 \pm 1,89$ & $5,29 \pm 3,19$ & $6,90 \pm 0,71$ & 0,192 \\
\hline
\end{tabular}

*Kruskall-Wallis; $p \leq 0,05$

Ao comparar somente atletas do sexo masculino segundo a categoria (Tabela 6), apenas no item condicionamento físico existe diferença estatística significativa entre as categorias, observada entre mirim $X$ infantil, infantil $X$ infanto, infantil $X$ juvenil.

Tabela 6 - Comparação dos Fatores Motivacionais dos

Participantes segundo Categoria - Gênero Masculino

\begin{tabular}{|c|c|c|c|c|c|c|}
\hline & \multirow{2}{*}{$\begin{array}{c}\text { Fatores } \\
\text { Motivacionais }\end{array}$} & \multicolumn{4}{|c|}{ Média e Desvio Padrão } & \multirow[b]{2}{*}{ Valor de $\mathrm{p}$} \\
\hline & & $\begin{array}{l}\text { Mirim } \\
(n=\mid 4)\end{array}$ & $\begin{array}{l}\text { Infantil } \\
(n=15)\end{array}$ & $\begin{array}{l}\text { Infanto } \\
(n=\mid I)\end{array}$ & $\begin{array}{l}\text { Juvenil } \\
(n=10)\end{array}$ & \\
\hline I & Status & $7,63 \pm 1,76$ & $5,97 \pm 1,90$ & $7,39 \pm 1,54$ & $7,66 \pm 1,79$ & 0,701 \\
\hline 2 & Cond. Físico & $7,62 \pm 2,13$ & $5,93 \pm 1,87^{¥}$ & $7,82 \pm 1,57$ & $8,13 \pm 1,34$ & $0,0 \mid 2 *$ \\
\hline 3 & Lib. de Energia & $6,67 \pm 2,03$ & $5,79 \pm 1,83$ & $5,95 \pm 2,04$ & $6,97 \pm 1,56$ & 0,430 \\
\hline 4 & Aperf. Técnico & $8,77 \pm 1,18$ & $7,92 \pm 1,76$ & $8,50 \pm 1,17$ & $8,33 \pm 1,73$ & 0,587 \\
\hline 5 & Afiliação & $7,80 \pm 1,51$ & $6,36 \pm 2,03$ & $6,44 \pm 1,72$ & $7,38 \pm 1,36$ & 0,088 \\
\hline 6 & Saúde & $8,3| \pm|, 5 \mid$ & $6,11 \pm 2,99$ & $7,73 \pm 2,22$ & $8,33 \pm 1,89$ & 0,148 \\
\hline 7 & Contexto & $6,87 \pm 2,13$ & $5,33 \pm 2,13$ & $3,64 \pm 1,71$ & $5,82 \pm 2,31$ & 0,237 \\
\hline
\end{tabular}

*Kruskall-Wallis; $p \leq 0,05$

$¥$ Mann-Whitney: mirim $x$ infantil; $p=0,018$ - infantil $x$ infanto; $p=0,009$ - infantil $\times$ juvenil; $p=0,003$ 
Ao comparar apenas atletas da categoria mirim segundo o gênero o fator afiliação apresentou diferença estatística, revelando ser as meninas mais motivadas por este fator que os meninos (Tabela 7).

Tabela 7 - Comparação dos Fatores Motivacionais segundo Gênero - Mirim

\begin{tabular}{l|llll}
\hline & Fatores Motivacionais & \multicolumn{2}{l}{ Mirim } & \\
\cline { 3 - 4 } & & Feminino $(\mathrm{n}=15)$ & $\begin{array}{l}\text { Masculino } \\
(\mathrm{n}=14)\end{array}$ & Valor de $p$ \\
1 & Status & $8,02 \pm 1,95$ & $7,63 \pm 1,76$ & 0,354 \\
2 & Condicionamento Físico & $7,96 \pm 1,86$ & $7,62 \pm 2,13$ & 0,847 \\
3 & Liberação de Energia & $7,86 \pm 1,43$ & $6,67 \pm 2,03$ & 0,112 \\
4 & Aperfeiçoamento Técnico & $9,52 \pm 0,47$ & $8,77 \pm 1,18$ & 0,123 \\
5 & Afiliação & $9,08 \pm 0,69$ & $7,80 \pm 1,51$ & $0,009 *$ \\
6 & Saúde & $8,42 \pm 2,03$ & $8,31 \pm 1,51$ & 0,683 \\
\hline 7 & Contexto & $7,31 \pm 1,72$ & $6,87 \pm 2,13$ & 0,533 \\
\hline
\end{tabular}

*Mann- Whitney Test; $p \leq 0,05$

Em relação aos atletas da categoria infantil, em todos os fatores, as meninas são mais motivadas quando comparadas aos meninos (Tabela 8).

Tabela 8 - Comparação dos Fatores Motivacionais segundo Gênero - Infantil

\begin{tabular}{|c|c|c|c|c|}
\hline & \multirow{2}{*}{ Fatores Motivacionais } & \multicolumn{2}{|l|}{ Infantil } & \multirow[b]{2}{*}{ Valor de $\mathrm{p}$} \\
\hline & & $\begin{array}{l}\text { Feminino } \\
(n=\mid 8)\end{array}$ & $\begin{array}{l}\text { Masculino } \\
(n=\mid 5)\end{array}$ & \\
\hline I & Status & $8,33 \pm 1,62$ & $5,97 \pm 1,90$ & $0,046^{*}$ \\
\hline 2 & Condicionamento Físico & $8,67 \pm 1,45$ & $5,93 \pm 1,87$ & $0,000 *$ \\
\hline 3 & Liberação de Energia & $7,99 \pm 1,86$ & $5,79 \pm 1,83$ & $0,00 I^{*}$ \\
\hline 4 & Aperf. Técnico & $9,31 \pm 0,91$ & $7,92 \pm 1,76$ & $0,0 \mid 8^{*}$ \\
\hline 5 & Afiliação & $8,91 \pm 1,48$ & $6,36 \pm 2,03$ & $0,000^{*}$ \\
\hline 6 & Saúde & $8,54 \pm 1,84$ & $6,11 \pm 2,99$ & $0,012^{*}$ \\
\hline 7 & Contexto & $7,69 \pm 1,89$ & $5,33 \pm 2,13$ & $0,002^{*}$ \\
\hline
\end{tabular}

*Mann- Whitney Test; $p \leq 0,05$

Comparando atletas da categoria infanto segundo o sexo, as atletas se mostraram mais motivadas em relação ao fator Afiliação do que os atletas (Tabela 9). 
Tabela 9 - Comparação dos Fatores Motivacionais segundo Gênero - Infanto

\begin{tabular}{|c|c|c|c|c|}
\hline & \multirow{2}{*}{ Fatores Motivacionais } & \multicolumn{2}{|l|}{ Infanto } & \multirow[b]{2}{*}{ Valor de $\mathrm{p}$} \\
\hline & & $\begin{array}{l}\text { Feminino } \\
(n=\mid I)\end{array}$ & $\begin{array}{l}\text { Masculino } \\
(n=\mid I)\end{array}$ & \\
\hline I & Status & $6,27 \pm 2,50$ & $7,39 \pm 1,54$ & 0,309 \\
\hline 2 & Condicionamento Físico & $7,39 \pm 2,37$ & $7,82 \pm 1,57$ & 0,847 \\
\hline 3 & Liberação de Energia & $6,29 \pm 2,68$ & $5,95 \pm 2,04$ & 0,652 \\
\hline 4 & Aperfeiçoamento Técnico & $7,95 \pm 1,93$ & $8,50 \pm 1,17$ & 0,699 \\
\hline 5 & Afiliação & $7,91 \pm 1,81$ & $6,44 \pm 1,72$ & $0,034 *$ \\
\hline 6 & Saúde & $7,30 \pm 2,58$ & $7,73 \pm 2,22$ & 0,748 \\
\hline 7 & Contexto & $5,29 \pm 3,19$ & $3,64 \pm 1,71$ & 0,949 \\
\hline
\end{tabular}

*Mann- Whitney Test; $p \leq 0,05$

Todos os fatores motivam da mesma forma os atletas da categoria juvenil, independentemente de serem do gênero masculino ou feminino (Tabela I0).

Tabela 10 - Comparação dos Fatores Motivacionais segundo Gênero - Juvenil

\begin{tabular}{l|llll}
\hline & \multirow{3}{*}{ Fatores Motivacionais } & \multicolumn{2}{l}{ Juvenil } & \\
\cline { 3 - 4 } & & $\begin{array}{l}\text { Feminino } \\
(\mathrm{n}=02)\end{array}$ & $\begin{array}{l}\text { Masculino } \\
(\mathrm{n}=10)\end{array}$ & \\
& & $8,21 \pm 0,91$ & $7,66 \pm 1,79$ & 0,590 \\
1 & Status & $7,00 \pm 0,94$ & $8,13 \pm 1,34$ & 0,364 \\
2 & Condicionamento Físico & \\
3 & Liberação de Energia & $8,25 \pm 0,35$ & $6,97 \pm 1,56$ & 0,485 \\
4 & Aperfeiçoamento Técnico & $9,63 \pm 0,18$ & $8,33 \pm 1,73$ & 0,182 \\
5 & Afiliação & $7,50 \pm 1,56$ & $7,38 \pm 1,36$ & 0,909 \\
6 & Saúde & $8,50 \pm 1,18$ & $8,33 \pm 1,89$ & 0,909 \\
\hline 7 & Contexto & $6,90 \pm 0,71$ & $5,82 \pm 2,31$ & 0,758 \\
\hline
\end{tabular}

*Mann- Whitney Test; $p \leq 0,05$

\section{DISCUSSÃO}

Os sete fatores motivacionais propostos por Barroso (2007), dependendo da média obtida pelas respostas das perguntas que compõem um mesmo fator motivacional, podem ser classificados em nada importante $(0$ a 0,99$)$, pouco importante (I a 3,99), importante $(4$ a 6,99), muito importante (7 a 9,99) e totalmente importante (10). Os participantes do estudo atribuíram o conceito "muito importante" para todos os fatores motivacionais (Tabela 2). Este resultado indica que os atletas 
pesquisados eram altamente motivados para a prática do vôlei de quadra competitivo. Lizieiro (2008), estudando adolescentes federados da modalidade futebol, na cidade de Campo Grande/MS, constatou que seus participantes também atribuiram conceito "muito importante" para todos estes fatores.

O fator considerado de maior motivação entre os atletas, independentemente da categoria ou gênero, foi aperfeiçoamento técnico, de acordo com as Tabelas 2, 3 e 4 . Este resultado tem ligação com o fato de todos os participantes da pesquisa estarem envolvidos com o esporte na perspectiva de competição/rendimento. Esse resultado corrobora com os achados de Gaya e Cardoso (1998), que ao pesquisar os fatores motivacionais de crianças e adolescentes em clubes esportivos encontraram os motivos relacionados à competência desportiva os mais importantes nestes locais de prática. Estudos de Paim (200 I) e de Gürbüz et al. (2007) ao analisarem especificamente fatores motivacionais em adolescentes praticantes de futebol também verificaram competência desportiva como fator determinante para a prática desse esporte. O estudo de Barroso (2007) com atletas universitários das modalidades de basquete, futsal, handebol e voleibol, também corrobora com esse entendimento, apresentando o aperfeiçoamento técnico como fator de maior motivação.

Em contrapartida, estudos de Paim e Pereira (2004) e Interdonato et al. (2008) determinaram ser saúde o principal fator motivacional. Esses autores afirmam que a difusão da prática de atividades físicas como agente propiciador de saúde, influenciando a melhora da qualidade de vida, contribui para os índices motivacionais encontrados neste fator. No presente estudo, este item foi o segundo na ordem de importância entre os sete fatores analisados, mostrando também ter grande relevância para os atletas. Rosolen (2006), ao estudar atletas universitárias de futsal feminino, encontrou a seguinte ordem de motivação para os fatores motivacionais: jogar em equipe; diversão; aprimoramento das habilidades; obter forma física; liberar energia; status; situacionais/outros e, finalmente, fazer amigos. É relevante ressaltar que, diferentemente do presente estudo, em que o aperfeiçoamento técnico é o mais motivante, o fator análogo aprimoramento das habilidades só foi identificado como o terceiro fator motivacional.

Na comparação dos fatores motivacionais dos participantes segundo gênero, detectou-se haver diferença estatística significativa em todos os fatores analisados, exceto nos itens Saúde e Status. É de grande valia ressaltar que em todos os itens em que há diferença entre os grupos, as atletas se sentem mais motivadas por esses fatores do que os atletas (Tabela 3). Entretanto, os resultados apresentados por Belmonte (2002) mostram uma tendência dos meninos a valorizarem mais as questões referentes ao fator "aspirações esportivas" do que as meninas. 
Os resultados da comparação dos fatores motivacionais segundo categoria demonstraram que atletas mirins, infantis, infantos ou juvenis se motivam da mesma forma. A única exceção foi no item afiliação, em que atletas mirins se motivam mais que os da categoria infanto ou juvenil. $\bigcirc$ item afiliação engloba questões relacionadas a estar com amigos/ pertencer a uma equipe. Gallahue e Ozmun (2003) afirmam que a necessidade de pertencer a um grupo é muito forte na adolescência, podendo ser um dos fatores primordiais para que jovens se envolvam com o esporte. Sendo a mirim a primeira categoria, aquela em que as crianças se conhecem e passam a formar um grupo, esse sentimento de pertencer a uma equipe parece ser mais significativo para motivar os atletas mirins que os das categorias infanto e juvenil.

Conforme foi constatado anteriormente, a categoria tem pouca influência em como os sete fatores motivacionais analisados influenciam os participantes. Ao comparar somente atletas do gênero feminino segundo categoria (Tabela 5) não houve diferença estatística significativa em nenhum fator. Entre atletas do sexo masculino (Tabela 6) houve diferença somente quando comparada a categoria infantil com as outras: mirim, infanto e juvenil. Destaca-se que apenas a categoria infantil masculino apresentou valor mais baixo atribuído a esse item, demonstrando ser uma peculiaridade desta categoria o fator condicionamento físico não ser tão motivante quanto é para as demais categorias.

Ainda é importante ressaltar que ao comparar os fatores segundo o gênero, levando em consideração somente a categoria mirim (Tabela 7) e a categoria infanto (Tabela 9), as meninas são diferentes dos meninos apenas no item afiliação, sugerindo que nessas categorias, estar entre amigos/pertencer a um grupo motiva mais as meninas do que os meninos a continuarem a praticar o vôlei competitivo.

Ao comparar os resultados da categoria infantil segundo o gênero (Tabela 8), constata-se que as meninas são mais motivadas em todos os fatores que os meninos. Já na categoria juvenil (Tabela 10) esses fatores influenciam da mesma forma os atletas, independentemente do sexo.

\section{CONCLUSÃO}

O estudo demonstrou que os atletas pesquisados estavam altamente motivados para a prática do vôlei de quadra competitivo, tendo em vista que foi atribuída classificação muito importante a todos os fatores motivacionais analisados.

Aperfeiçoamento técnico foi indicado pelos atletas como o fator de maior motivação, independentemente de sexo ou categoria. 
Houve diferença estatística significativa em todos os fatores analisados, exceto nos itens Saúde e Status, ao comparar os fatores motivacionais segundo gênero. Em todos os itens em que há diferença entre os grupos, as atletas se sentem mais motivadas por esses fatores do que os atletas.

Ao comparar os fatores motivacionais segundo categoria, verificou-se que não há diferenças estatísticas significativas. A única exceção foi no item afiliação, em que atletas mirins se motivam mais que os das categorias infanto e juvenil.

Recomenda-se que estudos futuros ampliem o público pesquisado para possibilitar novos estudos estatísticos que investiguem: I) a possibilidade de haver diferenças entre os sexos, sugerindo maior motivação das jovens atletas quando comparadas aos jovens atletas e 2) a possibilidade da categoria à qual o atleta pertence ter pouca influência na intensidade em que os sete fatores analisados os motivam.

\section{Motivation factors of young volleyball athletes}

ABSTRACT: This study aimed to identify how the motivational factors determine the permanence of young athletes in volleyball high level and compare these factors by gender and by category. Data collection was performed by applying the questionnaire Escala de Motivos para a Prática Esportiva (EMPE) to 96 young athletes from indoor volleyball (46 girls and 50 boys). The factors that most motivated the athletes to practice volleyball were: Skills Development, Health and Affiliation. Comparing of the motivational factors according to gender, except the factors health and status, the girls had higher motivation than the boys. Comparing motivational factors according to category, all items tested, except Affiliation, motivated the athletes the same way.

KEYWORD: Motivation; Young Athletes Trainning; Volleyball Indoor; young athletes.

\section{Factores motivacionales de jóvenes atletas de voley}

RESUMEN: El estudo se centra en los factores motivacionales para determinar la persistencia de los jóvenes atletas en el voleibol de alto nivel y compararlas en relación al género y categoría. Los datos fueron recolectados mediante la aplicación del cuestionario de la Escala de Motivos para a Esportiva (EMPE) a 96 jóvenes jugadores de voleibol de salón (46 niñas y 50 niños). Los factores que motivaron a los deportistas para practicar voleibol fueron: el desarrollo técnico, la salud y afiliación. En comparación con el género, con la excepción de los factores y el estado de salud, las ninãs atletas tuvieron una mayor motivación de los niños atletas. Al comparar los factores motivacionales en base a las variables utilizadas en el estudio, a exepción de la adhesión, motivaron a los individuos o jugadores a practicar el Voley de Salón de la misma manera.

PALABRAS CLAVE: Motivación; Formación Juvenil; Voley de Salón; jóvenes atletas. 


\section{REFERÊNCIAS}

ALVAREZ, E. et al. Dropout reasons in young Spanish athletes: relationship to gender, type of sport and level of competition. Journal of Sport Behaviour, Mobile, v. 29, p. 255-270, 2006.

BARROSO, M. Validação do Participation Motivation Questionnaire adaptado para determinar motivos de prática esportiva de adultos jovens brasileiros. 2007. I30f. Dissertação (Mestrado em Ciência do Movimento Humano) - Centro de Ciências da Saúde e do Esporte, Universidade do Estado de Santa Catarina, Florianópolis, 2007.

BARROSO, M. et al. Motivos de prática de esportes coletivos universitário em Santa Catarina. In: FÓRUM INTERNACIONAIS DE ESPORTES, 6., 2007, Florianópolis. Anais... Florianópolis: Uneposrtes, 2007. p. I I. I - I I.9 Disponível em: < http://www.unesporte.org.br/forum2007/ apresentacao_oral/I I_mario_luiz_barroso.pdf>. Acesso em: 19 mar. 2009.

BELMONTE, C. Fatores motivacionais para prática desportiva e suas relações com o gênero sexual. In: SALÃO DE INICIATIVA CIENTÍFICA, 2002, Porto Alegre. Anais... Porto Alegre: UFRGS, 2002. p. 508.

BENCK, R.; CASAL, H. Atribuições de causalidade para o sucesso e fracasso em diferentes modalidades esportivas. Lecturas: Educación Física Y Deportes, Buenos Aires, v. 10, n. 92 , 2006. Disponível em: <http://www.efdesportes.com/efd92/atrib.htm>. Acesso em: I9 maio 2009.

BERLEZE, A.; VIEIRA, L. F.; KREBS, R. J. Motivos que levam crianças para a prática de atividades motoras na escola. Revista da Educação Física/UEM, Maringá, v. I3, n. I, p. 99- I07, 2002. Disponível em: <http://periodicos.uem.br/ojs/index.php/RevEducFis/article/view/3747>. Acesso em: 04 abr. 2009.

DAVIDOFF, L. L. Introdução à psicologia. 3. ed. São Paulo: Makron Books, 2001 .

GALLAHUE, D.; OZMUN, J. Compreendendo o desenvolvimento motor de bebês, crianças, adolescentes e adultos. 2. ed. São Paulo: Phorte, 2003.

GAYA, A.; CARDOSO, M. Os fatores motivacionais para a prática esportiva e suas relações com o sexo, idade e níveis de desempenho esportivo. Perfil, Porto Alegre, ano 2, n. 2, 1998.

GÜRBÜZ, B; ALTINTAS, A.; ASCI, F. Participation motives of 9- I 5 years old Turkish soccer players. Journal of Sports Science and Medicine, Antalya/Turquia. v. 6, s. 10, p. 89-90, 2007. Disponível em: <http://www.jssm.org/suppls/I0/Suppl. I0.p88-9l.pdf>. Acesso em: I9 mar. 2009. 
INTERDONATO, G. C. et al. Fatores motivacionais de atletas para a prática esportiva. Motriz, Rio Claro, v. 14, n. I, p. 63-66, Jan./Mar. 2008. Disponível em: <http://cecemca.rc.unesp. br/ojs/index.php/motriz/article/viewFile//290/I 589>. Acesso em: 19 mar. 2009.

JONES, G. W.; MACKAY, K, S.; PETERS, D. M. Participation motivation in martial artists in the west midlands region of England. Journal of Sports Science and Medicine, Worcester/UK, n. 5, p. 28-34, 2006.

KNIJNIK, J.; GREGUOL, M.; SILENO, S. Motivação no esporte infanto-juvenil: uma discussão sobre razões de busca e abandono da prática esportiva entre crianças e adolescentes. Revista Instituto de Ciências da Saúde, São Paulo, v. 19, n. 01, p. 7-13, 2001.

LIZIERO, L. Fatores Motivacionais de Adolescentes praticantes de futebol em Clubes Campo Grande/MS. 2008. Monografia - Departamento de Educação Física, Universidade Federal do Mato Grosso do Sul, Campo Grande, 2008.

MAGILL, R. Aprendizagem motora: conceitos e aplicações. São Paulo: Edgard Blücher, 1984.

MOROUÇO, P. Avaliação dos factores psicológicos inerentes ao rendimento: estudo realizado em nadadores cadetes do Distrito de Leiria. Psicologia.com.pt: o portal dos psicólogos, Porto, p. I-20. Disponível em: <www.http://psicologia.com.pt>. Acesso em: 20 de ago. 2009.

PAIM, M. Motivos que levam os adolescentes a praticar o futebol. Lecturas Educación Física y Deportes, Buenos Aires, v. 7, n. 43, Dez. 200 I. Disponível em: <http://www.efdeportes. com/efd43/motivo.htm>. Acesso em: 27 mai. .2009.

PAIM, M.; PEREIRA, E. Fatores motivacionais dos adolescentes para prática de capoeira na escola. Motriz, Rio Claro, v. 10, n. 3, p. 159-166, Set./Dez. 2004. Disponível em: <http:// www.rc.unesp.br/ib/efisica/motriz/I0n3/09CCP_hp.pdf>. Acesso em: 19 mar. 2009.

ROSOLEN, M.; VOSER, R. C.; HERNANDEZ, J.A. E. A motivação para o esporte em atletas universitárias de futsal feminino. Futsal Brasil: O Portal do Futsal Mundial. Porto Alegre. Disponível em: <http://www.futsalbrasil.com.br/artigos/artigo.php?cd_artigo=143>. Acesso em 14 jul. 2009.

SAMULSKI, D. M. Psicologia do Esporte: manual para a Educação Física, Psicologia e Fisioterapia. São Paulo: Manole, 2002.

WEINBERG. R.; GOULD, B. Foundations of sport and exercise psychologist. Champaign: Human Kinetics Publishers, 2001 . 
Recebido: 13 abr. 2010 Aprovado: 15 de nov. 2010

Endereço para correspondência: Lívia Tavares da Silva Campos Rua Gustavo Sampaio, n. 676, apto. 1208, Leme Rio de Janeiro - RJ CEP: 22010-010 